Research Report

\title{
SEMANTIC BLINDNESS: \\ Repeated Concepts Are Difficult to Encode and Recall Under Time Pressure
}

\author{
Donald G. MacKay and Michelle D. Miller \\ University of California, Los Angeles
}

Abstract-This study demonstrates a recently predicted cognitive phenomenon known as semantic blindness, an inhibitory effect attributable to concept repetition in the serial recall of rapidly presented sentences. Proficient bilinguals read mixed, Spanish-English sentences, each including a target and a pretarget word. Targets and pretargets were related in three ways: They were identical (e.g., like-like), semantically identical across languages (e.g., gusta-like), and nonidentical within or across languages (e.g., read-like). Equivalent repetition blindness was found for targets with identical and semantically identical pretargets, indicating that repetition deficits were occurring solely at the semantic level, rather than at orthographic or phonological levels.

Repetition blindness (RB) refers to the inability to detect or recall a repeated word in rapid serial visual presentation (RSVP; see, e.g., Bavelier \& Potter, 1992). The present article asks two questions about the level at which RB occurs: Is orthographic or phonological identity always necessary for occurrence of RB? Or is semantic identity sometimes sufficient to cause RB, which in this hypothetical case could be called semantic blindness (SB)? Both questions are relevant to a recently developed theory of repetition deafness and blindness (Miller \& MacKay, 1991; this issue, pp. 47-51) that predicts the existence of SB and semantic deafness. Demonstrating these phenomena is important not just to test this theory, but also to determine whether words can be registered in short-term memory via semantic as well as visual, orthographic, and phonological codes (see Bavelier \& Potter, 1992).

Address correspondence to Donald G. MacKay, Psychology Department, University of California, Los Angeles, Los Angeles, CA 90024.
Bavelier and Potter (1992) argued that visual characteristics such as spatial location, visual form, and visual format are irrelevant to RB. Identical spatial location is irrelevant because RB is equivalent whether subjects read sentences word by word and left to right or by RSVP (Kanwisher, 1987). Identical visual form and format are likewise irrelevant because $R B$ is unaffected when pretarget and target differ in case, as in "They wanted more than NINE but nine was all they got" (pretarget and target italicized; Bavelier \& Potter, 1992; Kanwisher, 1987), or in visual format, as in "They wanted more than 9, but nine was all they got." Because 9 and nine share no visual features, not even abstract ones, Bavelier and Potter suggested that phonological identity suffices to induce $\mathrm{RB}$, and that words are initially registered only phonologically in short-term memory. Despite the conceptual identity of 9 and nine, Bavelier and Potter rejected semantics as an initial code because Kanwisher and Potter (1990) had found that "noun synonyms" as in Sentences 1 and 2 did not induce SB:

1. The company's new toxin might poison people accidentally.

2. The new students worked with pupils who might help them.

From this failure to find SB, Kanwisher and Potter concluded that "repetition blindness occurs at a level of processing prior to the attainment of conceptual representations"' (pp. 42-43; see also Potter, 1993).

We had several reasons for reopening the search for SB: One is that two words in the same language are never completely identical in meaning (Lyons, 1968; MacKay, 1973). To illustrate, note that poison and toxin differ in both lexical and connotative meaning in Sentence 1: Subjects undoubtedly comprehended to poison as an action harmful to health, but could have comprehended toxin as beneficial to health, as in medical use of toxins to induce antibodies. Some of Kanwisher and Potter's (1990) synonyms also differed in reference as well as lexical and connotative meaning. For example, subjects reading Sentence 2 may have comprehended students and pupils as referring to different sets of people (referents). In short, if occurrence of SB requires complete conceptual identity, Kanwisher and Potter may have failed to find SB because their synonyms differed in lexical concept, connotation, and (sometimes) reference.

Another reason for reopening the search for SB is that identical lexical nodes play a central role in RB in the node structure theory (NST; Miller \& MacKay, 1991, this issue). And whereas within-language synonyms involve different lexical nodes, only one lexical node underlies words that refer to familiar objects or concepts in the two languages of proficient bilinguals (MacKay, 1982). In the following sentence, for example, horses and its Spanish translation equivalent, caballos, share a single lexical node and are identical in connotation and reference: Both words refer with similar connotative import to the same object class.

3. They saw horses, but caballos were prohibitir to enter. (SB repeated-target condition)

The NST therefore predicts that proficient bilinguals will exhibit SB: For example, they will encode and recall caballos less often when the reference and meaning of its pretarget are identical (as in Sentence 3) than when they are different (as in Sentence 4).

4. They saw sheep, but caballos were prohibitir to enter. (SB unrepeated-target condition)

Because horses and caballos differ in orthography and phonology, such an outcome can be explained only in terms of 
shared lexical or referential units (the SB hypothesis).

Under the RB hypothesis, the word itself (its phonology or orthography) must be identical for RB to occur. The $\mathrm{RB}$ hypothesis predicts that encoding and recall of a target such as caballos will be much less likely in Sentence 5, where its pretarget (caballos) is identical, than in Sentences 3, 4, and 6, where pretargets differ from caballos in phonology and orthography.

5. They saw caballos, but caballos were prohibitir to enter. (RB repeated-target condition)

6. They saw oveja, but caballos were prohibitir to enter. (RB unrepeated-target condition)

\section{METHOD}

To test these hypotheses, we asked 48 highly fluent Spanish-English bilinguals (see Appendix 1) to read and immediately recall 32 RSVP strings: 16 fillers randomly interspersed among 16 experimental sentences (e.g., Sentences 3-6; see Appendix 1 for rules of construction). Eight fillers were ungrammatical (e.g., Sentence 7), and 8 were normal Spanish or English sentences without code switches (e.g., Sentence 8). Fillers ensured that subjects would not come to focus on code switching or target repetition, and could sometimes expect ungrammaticality, as might result from SB or RB.

7. When we went a la it was very crowded. (ungrammatical filler)

8. When we went to the store it was very crowded. (normal filler)

Experimental materials were presented at either 70 or $90 \mathrm{~ms} /$ word, with one or two short words separating repeated words (see Appendix 1 for details and rationale). Subjects were informed that words would appear one at a time at the center of the screen, speeded up to varying degrees, and that they were to immediately recall the words aloud and in correct order. Subjects were told that many strings would contain a mixture of Spanish and English words, and that if they could not remember a word or its language, they should guess. Subjects were warned that some strings might seem strange or ungrammatical, but that they should try to repeat strings verbatim without paraphrasing.

\section{RESULTS}

Percentages of correct target report (see Appendix 2) are 43 and 34 for unrepeated and repeated targets, respectively, in the SB (across-language) condition and 42 and 32 in the RB (withinlanguage) condition, signifying equivalent magnitudes of blindness. An analysis of variance (ANOVA) showed a main effect of repetition on number of correct target reports $(F[1,42]=6.87, p<.01)$, but no interaction between the two types of target repetition $(F[1,42]=0.93, p=$ .93).

\section{DISCUSSION}

Our results support the SB hypothesis and indicate that meaning is registered early in short-term memory: Because SB translation equivalents differed in phonology and orthography, the superior recall of unrepeated relative to repeated SB targets cannot be explained at orthographic or phonological levels. This SB effect suggests an alternate explanation for several earlier findings that have been attributed to phonological identity (e.g., Bavelier \& Potter's, 1992, 9-vs.nine effect) or to visual-orthographic identity (e.g., Kanwisher \& Potter's, 1990 , suffix-identity effect). These effects are now explainable in terms of shared reference or semantics rather than shared visual, orthographic, or phonological features. Superior recall of unrepeated relative to repeated targets can no longer be automatically attributed to nonsemantic factors.

Moreover, the equivalence of SB and RB under the present experimental conditions rules out effects of phonology or orthography in our data: To support a role for orthographic or phonological identity, the difference in recall of repeated versus unrepeated targets should have been larger for RB conditions than for SB conditions, contrary to the present results. This equivalence of SB and RB effects strongly supports the NST (Miller \& MacKay, 1991, this issue), according to which $\mathrm{RB}$ and $\mathrm{SB}$ share the same underlying basis: A single node or set of nodes represents a lexical concept in long-term memory, and sentence comprehension proceeds in part by forming connections from already existing lexical nodes to phrase-level nodes. For example, consider how a proficient German-English bilingual comprehends the sentence "They saw Pferde, but horses were verboten there" (see the nodes in Fig. 1). The single lexical node for the repeated concept (Pferde/horses) must quickly connect with two nodes, for the verb phrase "saw horses" and for the proposition "horses were forbidden." Lexical nodes for unrepeated concepts (e.g., they, saw, were, and verboten in Fig. 1) likewise become connected with phrase nodes. The difference is that connections from unrepeated concepts are one-to-one and can be formed in parallel, whereas connections from repeated concepts are one-tomany and must be formed in sequence, requiring added time (see Miller \& MacKay, this issue). Given time pressure, as in RSVP experiments, the first connection from a repeated concept may be formed successfully but the second may not, so that the second concept is

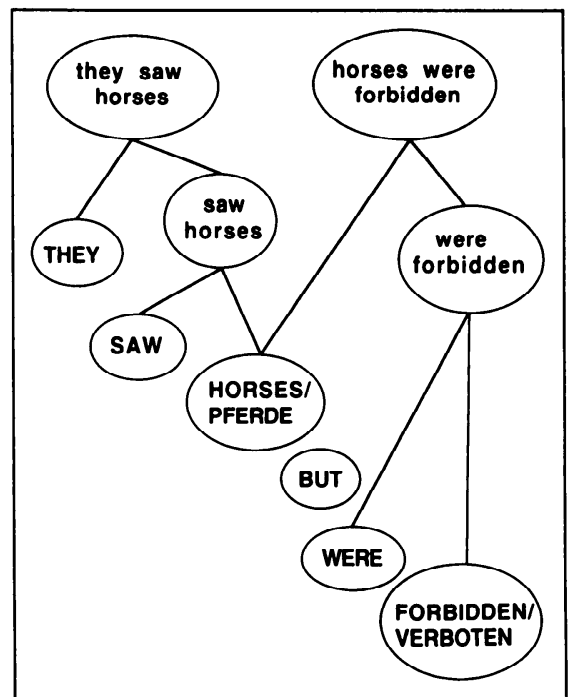

Fig. 1. Phrase nodes (lower case) and lexical nodes (capitals) for a proficient German-English bilingual comprehending aspects of the sentence "They saw Pferde, but horses were verboten there." Note that a single lexical node codes the repeated concept $P$ ferdel horses, the hypothetical basis for SB. Orthographic and phonological nodes are omitted from the figure. 
Semantic Blindness

unencoded and its phonology or orthography is unretrievable.

Principles whereby NST explains the equivalence of $\mathrm{SB}$ and $\mathrm{RB}$ in the present experiment also apply at other levels to explain demonstrations of RB without involvement of lexical nodes. An interesting example is the demonstration (MacKay, 1969) of RB for misspelled words in sentences. Repeated-letter misspellings (e.g., elderdly) were harder to detect than unrepeated-letter misspellings (e.g., elderkly). Like RB for RSVP words (Kanwisher, 1987), this repeatedletter effect reflects an encoding problem that diminishes with lag or degree of separation of the repeated letters. Interestingly, analogous effects occur in memory: Given perception of a misspelling, repeated-letter misspellings were more poorly recalled than unrepeated-letter misspellings (MacKay, 1969). Neither misspelling effect depended on crosssentence or cross-item interference (unlike Ranschburg inhibition; see Jahnke, 1972), but both effects involved relatively long processing times: The subjects read sentences at $96 \mathrm{~ms} / \mathrm{letter}$ or $116 \mathrm{~ms} /$ phoneme on average, considerably slower than rates at which RSVP RB has been demonstrated (about 25 to $60 \mathrm{~ms}$ (phoneme). Parametric studies of rate therefore seem warranted to determine whether rate limits of RB (see Kanwisher, 1987) are item- or task-specific.

Three other clear instances of RB without involvement of lexical nodes are
RB for homonym pairs (e.g., like [verb] and like [preposition]), for homograph pairs (e.g., bass [fish] and bass [male voice]), and for homophone pairs (e.g., won and one; Bavelier \& Potter, 1992; Kanwisher \& Potter, 1990). Because differing lexical nodes underlie samespelling or same-sounding words that differ in meaning, these results argue for the existence of two types of blindness (semantic and orthographic-phonological).

Acknowledgments-Aspects of this report were presented at UCLA and UC Berkeley in October 1992 and at the 33rd Annual Meeting of the Psychonomic Society, St. Louis, November 1992. Support from National Institute on Aging Grant 1 R01 AG 09755 to Donald G. MacKay and a National Science Foundation Graduate Fellowship to Michelle D. Miller is gratefully acknowledged. We thank Lise Abrams for setting up our computer program, Nancy Barba for creating the Spanish-English translations, Sarah Schuster for running subjects, and Deborah Burke for generously lending GenPrime and other equipment essential for this study, and for helpful comments on an earlier version of this article.

\section{REFERENCES}

Banks, W.P., Burke, D.M., Krajicek, D., \& Whet stone, T. (1990). Psychology lab: Developing Macintosh courseware for introductory psychology classes. Unpublished booklet, Psy- chology Department, Pomona College, Claremont, CA.

Bavelier, D., \& Potter, M.C. (1992). Visual and phonological codes in repetition blindness. Journal of Experimental Psychology: Human Perception and Performance, 18, 134-147.

Jahnke, J.C. (1972). The effects of intraserial and interserial repetition on recall. Journal of Verbal Learning and Verbal Behavior, 11, 706716 .

Kanwisher, N.G. (1987). Repetition blindness: Type recognition without token individuation. Cognition, 27, 117-143.

Kanwisher, N.G., \& Potter, M.C. (1990). Repetition blindness: Levels of processing. Journal of Experimental Psychology: Human Perception and Performance, 16, 30-47.

Lyons, J. (1968). Theoretical linguistics. Cambridge, England: Cambridge University Press.

MacKay, D.G. (1969). The repeated letter effect in the misspellings of dysgraphics and normals. Perception \& Psychophysics, 5, 102-106.

$\rightarrow$ MacKay, D.G. (1973). Complexity in output systems: Evidence from behavioral hybrids. American Journal of Psychology, 86, 785-806.

MacKay, D.G. (1982). The problems of flexibility, fluency, and speed-accuracy trade-off in skilled behavior. Psychological Review, 89, 483-506.

MacKay, D.G., \& Miller, M. (1992a, November). Repetition deafness and blindness: Rapidly presented repeated concepts are difficult to encode. Paper presented at the 33rd Annual Meeting of the Psychonomic Society, St. Louis.

MacKay, D.G., \& Miller, M. (1992b, April). The repetition deafness phenomenon in young and older adults. Paper presented at the 4th Biennial Cognitive Aging Conference, Atlanta.

Miller, M., \& MacKay, D.G. (1991, November). Repetition blindness and deafness: New data and theory. Paper presented at the 32nd Annual Meeting of the Psychonomic Society, San Francisco.

Potter, M.C. (1993). Very short-term conceptual memory. Memory \& Cognition, 21, 156-161.

(RECEIVED 12/28/92; REVISION ACCEPTED 5/26/93)

\section{APPENDIX 1: METHODOLOGICAL DETAILS}

\section{Subjects}

Subjects were 19 males and 29 females (46 right-handed, 2 left-handed; mean age $=24.8$ years; $S D=8.33$ ) with normal (corrected) vision. Most were recruited for pay (\$7) from ads in the University of California, Los Angeles (UCLA), student newspaper; some were graduate students in Spanish at UCLA; and some volunteered for partial course credit in UCLA psychology classes. Subjects reported speaking Spanish longer than English ( $M=21$ years vs. 19 years), but using a 5-point scale, they rated their fluency slightly higher for English than Spanish $(M=3.9$ vs. 3.3). Thirty-five $(73 \%)$ listed their firstlearned language as Spanish, 11 (23\%) listed it as English, and 1 each listed it as Chinese and Polish.

\section{Experimental Sentences}

There were 128 experimental sentences (mean length $=10$ words, 5 in Spanish, 5 in English) formed by code switching within 16 English prototype sentences (adapted from MacKay \& Miller, 1992a). A typical prototype sentence is shown in Sentence 9 (unrepeated pretarget in parentheses), together with its eight code-switching translations in Sentences 10 through 17. Targets and pretargets are italicized and labeled as to language and repetition condition. For example, Sentence 13 is an SB sentence with a Spanish pretarget that is completely different from the
English target and is labeled "SB unrepeatedtarget condition: Spanish-English." Similarly, Sentence 17 is an RB sentence with a repeated English target and is labeled " $R B$ repeated-target condition: English-English."

\section{Example prototype sentence}

Her son (kids), my son, and his daughter go to school together.

10. SB repeated-target condition: EnglishSpanish

Her son, mi hijo, y his hija van a la escuela together.

11. SB repeated-target condition: SpanishEnglish

Su hijo, my son, and su daughter go to school juntos. 
12. SB unrepeated-target condition: EnglishSpanish

Her kids, mi hijo, y his hija van a la escuela together.

13. SB unrepeated-target condition: SpanishEnglish

Sus niños, my son, and su daughter go to school juntos.

14. RB unrepeated-target condition: Spanish-Spanish

Her niños, mi hijo, y his hija van a la escuela together.

15. RB unrepeated-target condition: EnglishEnglish

Sus kids, my son, and su daughter go to school juntos.

16. RB repeated-target condition: SpanishSpanish

Her hijo, mi hijo, y his hija van a la escuela together.

17. RB repeated-target condition: EnglishEnglish

Su son, my son, and su daughter go to school juntos.

As in Sentences 10 through 17, targets in repeated-and unrepeated-target versions of SB and RB sentences were identical: Only the pretarget differed. Also, as in Sentences 12 through 15 , pretargets in unrepeated-target versions of $S B$ and $R B$ sentences were translation equivalents. Targets and pretargets

Each trial was scored for correct inclusion of the pretarget and target in the subject's response. We then determined the conditional measure of recall, which we believe most accurately represents the magnitude of repetition deficits (MacKay \& Miller, 1992b). For conditional measures, repeated and unrepeated targets count as correct only if their pretargets are also recalled. Without recall of the pretarget, data for that trial are discarded.

Subjects recalled the target concept cor- never occurred first or last in a sentence, were always high in frequency, and, when unrepeated, differed in phonology, orthography, and visual shape in both Spanish and English. The eight versions of a prototype were all grammatically acceptable and virtually identical in syntax, and were counterbalanced across subjects.

On average, words in a sentence occurred in 10 RSVP frames or simultaneous displays. In the few cases where translation demanded more than one word per frame, corresponding concepts were identically framed. For example, two conceptually equivalent frames represented the translation equivalents "to play ball" and "jugar a la pelota," namely, /to play/ball/ and /jugar/a la pelota/. The number of code switches per sentence and samelanguage words per switch varied within and across the 16 sets of 8 sentences. Any given content word never occurred in more than 1 of the 16 prototype sentences.

\section{Procedure and Design}

A general-purpose program (GenPrime; Banks, Burke, Krajicek, \& Whetstone, 1990) controlled stimulus presentation to the monitor of a Macintosh Plus computer, beginning with a 200-ms warning, "Get ready for the next sentence." A string of question marks

\section{APPENDIX 2: ANALYTIC DETAILS}

rectly, but in the wrong language, on 14 trials, 7 with repeated targets and 7 with unrepeated targets, as in Sentence 18, where the target, $t w o$, was misrecalled as its conceptual equivalent, dos. Identical $p$ values and degrees of repetition deficit resulted when these 14 trials (1.8\% of the conditional data) were excluded in a separate analysis. Similar semantic errors also occur in single-language RB experiments, but likewise too rarely to discount RB as a phenomenon. An example (from MacKay \& Miller, 1992a) is Sentence 19, in which a pro-
(??????) followed each stimulus, calling for verbal recall. Subject output was recorded using a cassette recorder, but was also transcribed "on line" by the experimenter. After generating a response, the subject pressed the space bar to begin the next trial.

Each subject saw four SB sentences (target repetition across languages), four RB sentences (target repetition within languages), plus corresponding unrepeated-target versions for the remaining eight sentences, with $\mathrm{SB}$ and $\mathrm{RB}$ versions and language of the target and pretarget counterbalanced across subjects. Stimulus order varied at random across subjects except that average serial position for repeated and unrepeated targets was held constant. Presentation rate varied orthogonally with type of experimental stimulus, with four repeated-target sentences and four unrepeated-target sentences presented at each rate (i.e., $70 \mathrm{~ms} /$ word and $90 \mathrm{~ms} /$ word) for each subject. The 16 filler sentences were presented at fixed rates, either $50,70,90$, or 130 $\mathrm{ms} /$ word, with 4 at each rate. Each session began with four representative practice sentences presented in different orders and rates, counterbalanced across subjects. We chose 70 and $90 \mathrm{~ms} /$ word as rates on the basis of pilot data indicating correct target recall in the $50 \%$ range, thereby avoiding floor or ceiling effects that could distort the relative degree of SB versus $R B$.

noun was substituted for the repeated target (see also Sentence 20).

18. I vivo at five ocho two Iglesia Calle Misrecalled as "I vivo at five ocho dos Iglesia Calle" (cross-language semantic error) 19. We buy fruit when fruit is in season Misrecalled as "We buy fruit when it is in season" (within-language semantic error) 20. We buy juice when fruit is in season Misrecalled as "We buy fruit when juice is in season" (within-language semantic error) 